\title{
Polarimetry of sunspot penumbrae with high spatial resolution
}

\author{
N. Bello González ${ }^{1}$, O. V. Okunev ${ }^{1,2}$, I. Domínguez Cerdeña ${ }^{1}$, F. Kneer ${ }^{1}$, and K. G. Puschmann ${ }^{1}$ \\ 1 Universitäts-Sternwarte, Geismarlandstr. 11, 37083 Göttingen, Germany \\ e-mail: nazaret@uni-sw.gwdg.de \\ 2 Central Astronomical Observatory at Pulkovo, 196140 St. Petersburg, Russia
}

Received 13 September 2004 / Accepted 10 December 2004

\begin{abstract}
We present two-dimensional high-spatial-resolution spectropolarimetric observations of sunspot penumbrae. They were obtained in April 2002 and May 2003 with the "Göttingen" Fabry-Pérot spectrometer at the Vacuum Tower Telescope at the Observatorio del Teide (Tenerife). Speckle methods were applied for image reconstruction which resulted in a spatial resolution of 0 . 5 in the magnetograms of the penumbrae. We analysed Stokes $I$ and $V$ profiles of the Fe II $6149 \AA$ line, which exhibits no instrumental Stokes $Q / U \rightarrow V$ crosstalk, and of the Fe I line pair at $6302 \AA$. The main results are the following: 1) on scales larger than 0 ' 5 , the intensity pattern of penumbrae stays the same in the continuum and core images of the $6301.5 \AA$ line, which stem from $0 \mathrm{~km}$ and $300 \mathrm{~km}$ (above $\tau_{5}=1$ ), respectively. Yet at scales of 0.5 and smaller the pattern in the two spectral features is clearly different. 2) On the limb side of sunspots the Evershed flow is carried by dark filaments and on the centre side by bright features and their somewhat weakened tails. We explain this with a picture in which the velocity of hot rising gas is best seen on the centre side, while on the limb side the horizontal outward and possibly downward flows are seen when the gas has cooled down. 3) The un-combed structure of the magnetic field is confirmed. On the limb side, the more horizontal fields coincide with dark fibrils or with diffuse intensity structures. Generally, the more horizontal fields are located at the positions of strong outflows. 4) Strong line-of-sight components of the magnetic fields are not found in bright filaments but in dark structures, somewhat displaced from the darkest parts. Their positions do not coincide with those of the strongest velocity fields. In general, our results are compatible with the picture of low lying flow channels coincident with the horizontal magnetic field, or possibly emerging and diving down into sub-photospheric layers, like a "sea serpent". Some further dynamic phenomena are discussed, which demonstrate the richness of processes in penumbrae, and reveal unexpected properties.
\end{abstract}

Key words. Sun: sunspots - Sun: magnetic fields - techniques: high angular resolution

\section{Introduction}

Sunspots, observed since their detection (in Europe) at the time of Galileo Galilei about 1610, have remained fascinating research subjects. Especially sunspot penumbrae, with their high structuring, have attracted much attention from which ample scientific work originated, both observational and theoretical. Recent observations with improved solar instrumentation have revealed that sunspot penumbrae possess much finer structures than was thought a few decades ago. Thus, we are still at the beginning of understanding penumbrae with their still enigmatic intensity pattern, their magnetic field structures, and the basic processes which cause the structuring and the Evershed flow (Evershed 1909). We refer the reader to reviews by Wiehr (1999), Schlichenmaier (2003), Solanki (2003) and Bellot Rubio (2004) which contain many relevant references.

Early studies like those of Beckers \& Schröter (1969) were already devoted to the variations of magnetic field strengths and inclinations, to the fluctuations of the Evershed flow and to the relations between magnetic fields, velocities and the filamentary intensity pattern. Degenhardt \& Wiehr (1991) found (azimuthal) fluctuations of field inclinations (with respect to the vertical) of $7^{\circ}<\gamma<13.5$. Solanki \& Montavon (1993) introduced the term "un-combed" magnetic field structure, i.e. that more or less horizontal flux ropes are embedded in a less inclined background field to generate the net circular polarisation. Title et al. (1993) also found azimuthal fluctuations of field inclinations and coined them "fluted penumbra". In their picture (see their Fig. 17) the Evershed flow occurs along essentially horizontal field structures with a tendency to coincide with relatively dark penumbral filaments. Lites et al. (1993) detected what they call "spines", i.e. radial magnetic field structures with definitely stronger fields and lower inclination than the fibrils next to them, but uncorrelated with brightness. Thus, the picture arose that the horizontal flux ropes possess weaker fields than the background field and that they carry the Evershed flow. Schlichenmaier \& Schmidt (2000) describe rising up-flow channels at different penumbra radii which bend outwards and submerge at the outer penumbra border. Radiative transfer inversion techniques (del Toro Iniesta et al. 1994) were applied by Westendorp Plaza et al. (2001a,b). They assumed a one-component atmosphere within 
Table 1. FPI spectrometer settings during the observations with dates, observed spectral line, exposure times, step widths of the spectrometer, its $F W H M$, number of spectral positions in one scan, number of frames taken at each wavelength position and achieved spatial resolution of the polarimetric data after reconstruction.

\begin{tabular}{cccccccc}
\hline \hline Date & Spectral line & $\begin{array}{c}\text { Exposure } \\
\text { time }(\mathrm{ms})\end{array}$ & $\begin{array}{c}\text { Step width } \\
(\mathrm{m} \AA)\end{array}$ & $\begin{array}{c}F W H M \\
(\mathrm{~m} \AA)\end{array}$ & $\begin{array}{c}\text { No. spectral } \\
\text { positions }\end{array}$ & $\begin{array}{c}\text { No. frames } \\
\text { per spectr. pos. }\end{array}$ & $\begin{array}{c}\text { Spatial } \\
\text { resolution }\end{array}$ \\
\hline 29.04 .2002 & Fe I 6301.5 + 6302.5 & 30 & 31.8 & 44 & 33 & 4 & 0.5 \\
02.05 .2003 & Fe II 6149.3 & 20 & 27.8 & 42 & 18 & 8 & $0.0^{\prime} 6$ \\
\hline
\end{tabular}

the observational resolution element and extended the finding of flow channels to magnetic flux ropes which are found to be tightly correlated with the flows. This picture was confirmed with two-component inversions by Bellot Rubio et al. (2003).

The relation of the Evershed flow to intensity and its height within the penumbral atmosphere is still under debate. Signatures that the velocities are concentrated in dark continuum filaments have been seen, among others, by Degenhardt \& Wiehr (1994), Rimmele (1995), Stanchfield et al. (1997) and Westendorp Plaza et al. (2001b). No clear correlation between intensities and velocities was found by Wiehr \& Stellmacher (1989), Lites et al. (1990) and by Hirzberger \& Kneer (2001). Rimmele (1995) and Stanchfield et al. (1997) found elevated flow channels. This is difficult to understand in view of the increase of line bisector shifts with depth in the atmosphere as shown by, e.g., Stellmacher \& Wiehr (1981), Balthasar et al. (1997) and Hirzberger \& Kneer (2001).

Similarly, no obvious correlation between brightness and magnetic field strength or field inclination could be established (see Schlichenmaier 2003, for a summary of the observational results). Flows and magnetic fields seem to be physically linked while the intensities as proxies for the temperature depend on the peculiarities of the position within the penumbrae, of the height and inclination of the flows (magnetic fields) and of the history of the flows and magnetic fields.

We mention as mechanisms driving the Evershed flow the siphon flow model proposed by Meyer \& Schmidt (1968) and further elaborated by Montesinos \& Thomas (1997) and the moving flux tube model by Schlichenmaier et al. (1998, see also the discussion in Schlichenmaier 2003). Especially the moving flux tube model suggests thin elongated magnetic fibrils with diameters of the order of $100 \mathrm{~km}$, i.e. substantially smaller than $1^{\prime \prime}$.

Thus, to understand sunspot penumbrae and their physical structure, observations and spectropolarimetric measurements with very high spatial resolution are needed. Scharmer et al. (2002) and Rouppe van der Voort et al. (2004) have presented high-resolution broadband observations from the new Swedish $1 \mathrm{~m}$ solar telescope on La Palma. After image reconstruction, penumbral grains and filamentary structure are seen at the diffraction limit of 0 . $^{\prime} 1$ as well as newly detected dark cores within penumbral filaments. Using also image reconstruction methods, Hirzberger \& Kneer (2001) obtained spectroscopic data with high spatial resolution in the magnetically non-split Fe I $5576 \AA$ Aine. The Freiburg group (Tritschler et al. 2004; Schlichenmaier et al. 2004), successfully applying adaptive optics to penumbra observations with the same non-split
Fe I line, reached a spatial resolution of 0.5 . The latter authors investigated the line shifts and the line asymmetries and found the observations compatible with low lying flow channels with up-flows at the inner foot-points and down-flows after some $4 "$.

In the present study, we continue the efforts to obtain spectroscopic data with high spatial resolution, and extend them to polarimetry of magnetically sensitive spectral lines. The use of a two-dimensional, scanning Fabry-Pérot filtergraph allows image reconstruction after the observations. The spatial resolution thus obtained is substantially better than $1^{\prime \prime}$, even for polarimetric data. The next section describes the observations and the data analysis. We present the fine structures of intensity, magnetic field and velocity together with their relations in Sect. 3. Section 4 concludes the paper.

\section{Observations and data analysis}

\subsection{Observations}

The observations were performed during various campaigns in 2002 and 2003 with the German Vacuum Tower Telescope (VTT) at the Observatorio del Teide (Tenerife). They were obtained with the "Göttingen" Fabry-Pérot interferometer (FPI; Bendlin et al. 1992; Bendlin \& Volkmer 1995). The optical setup described by Koschinsky et al. (2001, their Fig. 1) was used except that the Stokes $V$ polarimeter was moved from the position near an intermediate focus to a position directly in front of the narrow band detector (CCD2). The data sets are described in Table 1. Spectral lines formed at different heights in the penumbral atmosphere and with different sensitivity to magnetic fields were scanned to observe two sunspots located at slightly different heliocentric angles as given in Table 2:

- Fe II $6149 \AA$, Landé factor $g=1.33$ and a particular Zeeman splitting which makes Stokes $Q$ and $U$ zero. Thus the line is free of instrumental $Q, U \rightarrow V$ crosstalk. The observed sunspot, NOAA0346, was located near disc centre, at heliocentric angle $\theta=23^{\circ}$.

- Simultaneously Fe I $6301 \AA$ and Fe I $6302 \AA$ with Landé factors $g_{\text {eff }}=1.67$ and $g=2.5$, respectively, on the sunspot NOAA9919 near disc centre, at $\theta=20^{\circ}$.

The data provided by the FPI spectrometer consist of sequences of the order of 130-150 simultaneous two-dimensional (2D) broadband and narrow-band frames. The narrow-band images are grouped into 4-8 frames taken at each scanning (wavelength) position. One wavelength scan takes approximately $50 \mathrm{~s}$ including storage of the images onto hard disc. 
Table 2. Observed sunspots during the different campaigns with dates, denominations, heliocentric angle and the spectral lines in which they were observed.

\begin{tabular}{cccc}
\hline \hline Date & Sunspot & $\begin{array}{c}\text { Heliocentric } \\
\text { Angle }(\theta)\end{array}$ & Line ( $) ~$ \\
\hline 29.04 .02 & NOAA9919 & $20^{\circ}$ & Fe I 6301.5 \\
& & & + Fe I 6302.5 \\
02.05 .03 & NOAA0346 & $23^{\circ}$ & Fe II 6149 \\
\hline
\end{tabular}

The Stokes $V$ polarimeter splits the light transmitted by the FPI into two beams of right and left circularly polarised light, i.e. $\frac{1}{2}\left(I_{\lambda}+V_{\lambda}\right)$ and $\frac{1}{2}\left(I_{\lambda}-V_{\lambda}\right)$, respectively. The detector for the narrow-band frames collects both components and, thus, only half of the CCD detector of $385 \times 286$ pixel, with an image scale of $0.1 /$ pixel, is available for the field of view (FOV). To observe the sunspots, we proceeded to perform wavelength scans of one half of a sunspot and afterwards scans of the other half. Figure 3 gives an example.

\subsection{Image reconstruction}

The usual corrections were applied to the data: subtraction of the dark background, flat fielding and systematic effects introduced by the variation of the FPI transmission over the FOV (see Okunev 2004). The large number of broadband frames with short exposure times $(20-30 \mathrm{~ms})$ allows image reconstruction with speckle methods (de Boer \& Kneer 1992; de Boer 1996). The spectral ratio method (von der Lühe 1984) and the speckle masking method (Weigelt 1977) were applied. For the reconstruction of narrowband images the code developed by Janßen (2003) was used. It is based on the method proposed by Keller \& von der Lühe (1992; see also Krieg et al. 1999; and Hirzberger et al. 2001) to calculate the instantaneous optical transfer function from the observed and reconstructed broadband images. A least-squares calculation leads then, in Fourier space, to the estimates of the narrowband images $O_{\mathrm{n}}$

$O_{\mathrm{n}}(\boldsymbol{q})=H \cdot \frac{\sum I_{\mathrm{n}}(\boldsymbol{q}, t) I_{\mathrm{b}}^{*}(\boldsymbol{q}, t)}{\sum\left|I_{\mathrm{b}}(\boldsymbol{q}, t)\right|^{2}} \cdot O_{\mathrm{b}}(\boldsymbol{q})$,

where the summation runs over the frames taken at fixed wavelength positions. $I_{\mathrm{n}}$ and $I_{\mathrm{b}}$ are the narrow- and broadband images, respectively, $O_{\mathrm{b}}$ is the reconstructed broadband image. $\boldsymbol{q}$ denotes the 2D coordinates in Fourier space. An optimum filter $H$ is also included in Eq. (1).

The achieved spatial resolution is approximately 0.25 for the broadband images, i.e. close to the diffraction limit, and $0.5-0 . ' 6$, depending on the data set, for the narrowband images. Examples are shown in Fig. 1.

\subsection{Stokes I and V}

From the $\frac{1}{2}\left(I_{\lambda}+V_{\lambda}\right)$ and $\frac{1}{2}\left(I_{\lambda}-V_{\lambda}\right)$ components we obtain the Stokes $I$ and $V$ profiles by addition and subtraction respectively. This process must be done carefully. Displacements between the two components were eliminated with a sub-pixel interpolation. Differences of sensitivity of the left and right circular polarised channels were determined from line profiles of the average flat field frames which yielded normalisation factors applied to the sunspot data.

Two of the three methods described below to create 2D magnetograms are based on the $V$ profiles. We apply an algorithm, used by Okunev \& Kneer (2004), which fits each profile to two Gaussians (one per lobe) and a second order polynomial according to

$$
\begin{aligned}
Y(\lambda, \boldsymbol{a})= & a_{0} \mathrm{e}^{-\left(\lambda-a_{1}\right)^{2} /\left(2 a_{2}^{2}\right)}+a_{3} \mathrm{e}^{-\left(\lambda-a_{4}\right)^{2} /\left(2 a_{5}^{2}\right)} \\
& +a_{6}+a_{7} \lambda+a_{8} \lambda^{2}
\end{aligned}
$$

The parameters $\boldsymbol{a}=\left(a_{0}, \ldots, a_{8}\right)$ were initialised with characteristics of the observational profiles. Constraints were applied to avoid information from pixels with signal below the noise level or which does not fit to the expected Stokes $V$ shape with two lobes. We thus excluded more complicated $V$ profiles, e.g. with three lobes. From the fitted analytic $V$ profiles we extract the separation of the lobes and their amplitudes. The lower limit of detectable Stokes $V$ signals in one pixel in the FOV, i.e. the noise level, is $3 \ldots 5 \times 10^{-3} I_{\mathrm{c}}$, where $I_{\mathrm{c}}$ is the average penumbral continuum intensity.

\subsection{Magnetic field and velocity determination}

We do not have the full Stokes vectors available and the $I$ and $V$ profiles are broadened by Airy's FPI transmission function of the spectrometer. Thus, with the present data it is very difficult to apply inversion techniques for the determination of magnetic field strengths and field inclinations as functions of height in penumbra atmospheres. In the present high spatial resolution study we concentrate on the fluctuations of magnetic fields and on their relations to intensity and velocity and we allow errors in the field amplitudes. Forward modeling, i.e. assuming specific atmospheric, magnetic and flow models and examining the emergent profiles for compatibility with observations, will be deferred to a further investigation. Here, we apply three different methods for the measurement of magnetic fields as averages over the spatial resolution elements and over the signal formation heights along the LOS.

1) Weak Field Approximation - WFA. The weak field solution of the Unno-Rachkovski equations is (cf. Landi Degl'Innocenti 1992)

$V(\lambda)=-C B_{\mathrm{eff}} \frac{\mathrm{d} I_{\mathrm{m}}(\lambda)}{\mathrm{d} \lambda}$

with $C=4.67 \times 10^{-13} g_{\text {eff }} \lambda_{0}^{2}$ when $B_{\text {eff }}$ is measured in Gauss and wavelength in $\AA$. $I_{\mathrm{m}}(\lambda)$ is the un-split, i.e. to first order magnetically unaffected Stokes $I$ profile in the magnetic feature. The effective magnetic field strength is given by

$B_{\text {eff }}=\alpha \cos \gamma B$,

with a factor $\alpha<1$ accounting for stray light and $\gamma$ the angle between the magnetic field and the line of sight (LOS).

The WFA applies only for lines with moderate to small Landé factors $g_{\text {eff }}$, like Fe II $6149 \AA$ and Fe I $6301.5 \AA$, 

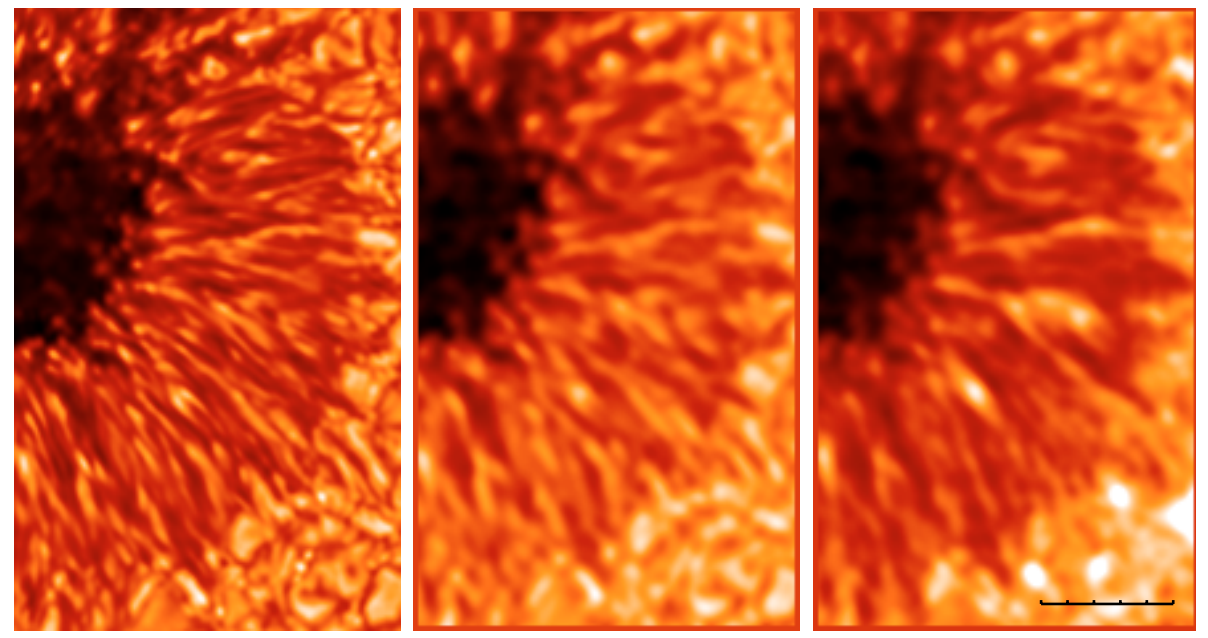

Fig. 1. Reconstructed images of the centre side of the sunspot NOAA9919 on 29.04.2002 in the $6301 \AA$ spectral range. From left to right: broadband image, narrowband image from the outer wings of Fe I $6301.5 \AA$ ("continuum" image, see Fig. 2), and narrowband image from the line core of the same line. The intensity of the line core image is re-scaled to emphasise the small-scale fluctuations. The distance of the tickmarks in the lower right corner corresponds to $1^{\prime \prime}$.

and in weak to moderate magnetic fields of strength $B<$ $1000 \mathrm{G}$ for the two lines. For stronger fields the determined strengths are systematically underestimated.

We assume that the resolution element is filled with magnetic features but contaminated with false light from scattering in the Earth's atmosphere and by the telescope optics. The amount of stray light has been determined in the case of the Fe II $6149 \AA$ line from measurements in the very dark umbral parts where this line should not occur.

2) Strong field regime - SFR. The formula for the Zeeman splitting $\left(\Delta \lambda_{B}\right)$ gives

$\Delta \lambda_{B}=4.67 \times 10^{-13} g_{\mathrm{eff}} \lambda_{0}^{2} B$

$B$ can be measured from the separation $\Delta \lambda_{B}$ of the $V$ extrema. It does not depend on the stray light and only weakly, through magneto-optical effects, on the angle $\gamma$. In the cases of the Fe I $6173.3 \AA$ and $6302.5 \AA$ lines, both with $g=2.5$, and for sufficiently strong magnetic fields $\left(\Delta \lambda_{B}>\Delta \lambda_{D}\right.$, or approximately $B>1500$ Gauss) the separation of the $V$ extrema starts to increase linearly with $B$ while the amplitude of Stokes $V$ becomes independent of $B$. For too low field strengths $B$ is measured systematically too large.

3) Centre of Gravity - COG. Semel (1967) and Rees \& Semel (1979) have shown that the wavelength separation of the COG of the profiles $\frac{1}{2}\left(I_{\lambda}+V_{\lambda}\right)$ and $\frac{1}{2}\left(I_{\lambda}-V_{\lambda}\right)$ gives the average field component along the LOS, i.e. with $B$ in Eq. (5) replaced by $B_{\text {eff }}$ from Eq. (4).

The velocities along the LOS were also determined with the COG method, as the average COG position of the $\frac{1}{2}(I+V)$ and $\frac{1}{2}(I-V)$ profiles. The average line position from the ambient non-spot area was taken as reference, i.e. $\bar{v}_{\text {gran, LOS }}=0$. This method of velocity measurement is applicable also in the nonmagnetic areas and, especially important for the Fe II $6149 \AA$ line, at positions in the FOV where the Stokes $V$ signal is below the noise even in the penumbra. Yet the method can give velocity values contaminated by scattered light. The latter has been taken into account for the Fe II $6149 \AA$ line from its umbral profile which we assume to be pure scattered light. For the $6302 \AA$ lines we estimated this effect by assuming that the false light reaches a reasonable level of $10 \%$ of the continuum intensity in the penumbra and that it carries an unshifted line profile from the quiet Sun. The line shift measurements turned out to be underestimated by $10 \%$.

\subsection{Instrumental polarisation}

Models and measurements of instrumental polarisation, i.e. Mueller matrix M, of the Donati Tower in Arcetri, a coelostat system as the VTT, have been published by Capitani et al. (1989). The instrumental crosstalk of the VTT has been measured early in the nineties by Hofmann (2001) and by Collados (1999). The Donati Tower and the VTT behave similarly. With the values from these works we made some estimates from model calculations of spectral lines in penumbral atmospheres with reasonable field strengths and inclination angles towards the LOS.

The $V \rightarrow I$ crosstalk, i.e. $\mathrm{M}_{14}$, amounts to approximately $1 \%$ (of $I$ ) and has only negligible influence on the velocity measurements. The main concern comes from the $Q, U \rightarrow V$ crosstalk, i.e. from the elements $\mathrm{M}_{42}$ and $\mathrm{M}_{43}$, which are of the order of 0.2-0.3 while the $Q$ and $U$ amplitudes are of the same order of 0.1 (relative to the continuum intensity $I_{\mathrm{c}}$ ) as the $V$ amplitudes. (For inclination angles of $90^{\circ}$, and neglecting magneto-optic effects, $Q$ and $U$ are at maximum while $V=0$.)

The Fe II $6149 \AA$ line, because of its specific Zeeman pattern, is free of $Q, U \rightarrow V$ crosstalk. For the $6302 \AA$ line pair we estimated the influence of the instrumental polarisation on the measurements in the following way: we calculated Stokes profiles emergent from the penumbra model by del Toro Iniesta et al. (1994) applying reasonable values of magnetic fields, of their inclination and gas velocities. From these profiles and the relevant elements of the instrumental Mueller matrix of the VTT the effects of the crosstalk on the measurements were calculated. The $V$ amplitude of Fe I $6301.5 \AA$ is changed by crosstalk by approximately $\pm 12 \%$, where the sign depends on the inclination angle. From Eq. (3) this is also the error of $B_{\text {eff }}$ when the WFA is applied. The COG determination of $B_{\text {eff }}$ for this line and for Fe I $6302.5 \AA$ gives values too small by $20 \%$, at most, because of crosstalk, while the distance of the $V$ extrema of the latter line is not affected. 


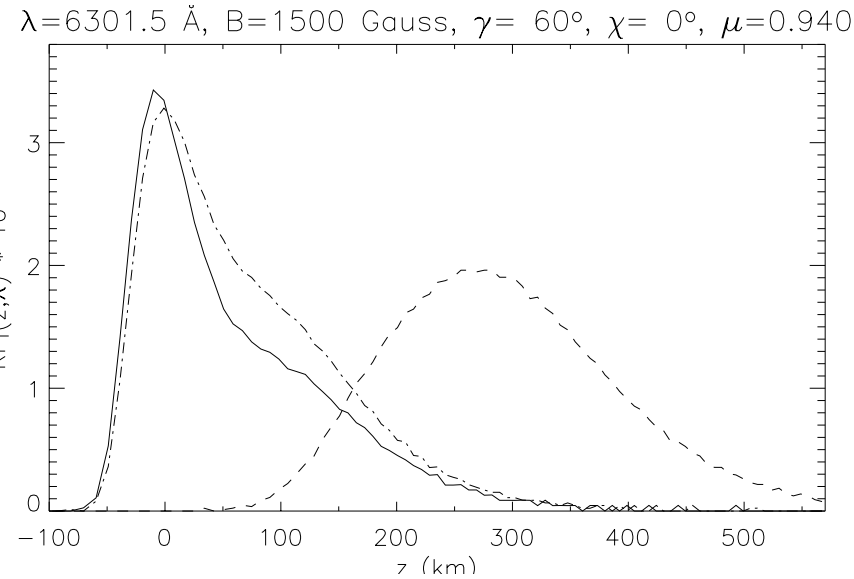

Fig. 2. Temperature response functions for Fe I 6301.5 A in the penumbra model of del Toro Iniesta et al. (1994). The units of the response functions are $\mathrm{erg} \mathrm{cm}^{-2} \mathrm{~s}^{-1} \mathrm{~Hz}^{-1} \mathrm{ster}^{-1} \mathrm{~K}^{-1} \mathrm{~km}^{-1}$. Solid curve: continuum response function $R F_{\mathrm{T}}$; dash-dotted: average from wavelengths in the outer wings; dashed: average from wavelengths around line centre. $\gamma$ is the angle between the magnetic field and the LOS, $\chi$ the azimuth of the magnetic field and $\mu=\cos \theta$.

\section{Results}

\subsection{Intensity fluctuations}

The intensity structure of the centre side part of the sunspot NOAA9919 at a heliocentric angle of $20^{\circ}$ is shown in Fig. 1. There the speckle-reconstructed broadband image exhibits the highest spatial resolution. To suppress noise, for the image from the outer wings of the $6301 \AA$ line an average of frames at line core $(\mathrm{lc})+256 \mathrm{~m} \AA$ and $\mathrm{lc}-224 \mathrm{~m} \AA$ is taken. Images from pure continuum are not available in our data. Yet for short we denote this latter image as "continuum" image. Likewise, the line core image is actually an average of the three images taken closest to lc.

We adopt intensity fluctuations as proxies for temperature fluctuations. To obtain information about their heights of formation we calculate temperature response functions (Mein 1971; see also Krieg et al. 1999). We use the average penumbra atmosphere model by del Toro Iniesta et al. (1994) and apply small temperature increases at specific heights requiring horizontal pressure equilibrium and assuming LTE for line formation. The differences of the intensity profiles from the perturbed and unperturbed atmospheres yield the response functions (cf. Eibe et al. 2001). Figure 2 depicts temperature response functions $R F_{\mathrm{T}}$ for several wavelengths in the $\mathrm{Fe} \mathrm{I} 6301.5 \AA$ line. A specific magnetic field configuration with constant field strength was chosen. We note that for typical penumbral field strengths within the range of 1000-2200 G and field inclinations the response functions for wavelengths near the centre of this line are similar. The line core fluctuations are formed in the height range of $150-400 \mathrm{~km}$ (above $\tau_{5}=1$ ). There exists a small overlap with the response functions for the wings of the line ("continuum" image). We emphasize that the temperature response functions near the line centre look different for strong magnetic fields $(>2000 \mathrm{G}$ ) oriented closely along the LOS. Then the Zeeman splitting becomes strong and the light at wavelengths around the line centre comes from deep layers of the atmosphere. Thus, the response functions in Fig. 2 are not designed for features in an umbra like, e.g., umbral dots, observed close to disc centre and with nearly vertical magnetic fields.

The penumbral small-scale fluctuations in the far-wing and line core images are of the same order, $20-30 \%$ peak to valley. Besides, they show a close correspondence, nearly one to one. We shall show below that the spatial resolution of these images is $0.45-0$. $^{\prime} 5$. Thus at this scale and larger the penumbral structures at deep photospheric levels are persistent up to heights of $300-400 \mathrm{~km}$. This agrees with the results obtained from inversions of filtergram data in the Fe I $5576 \AA$ line (del Toro Iniesta et al. 1994) and of Ca II K spectrograms (Rouppe van der Voort 2002).

Tests have shown that the speckle-reconstructed broadband images become nearly identical with the narrowband continuum images when the former are convolved with a Gaussian of $F W H M$ of 0.4 . The reason for the reduced spatial resolution of the narrowband images comes, presumably, from additional imaging through the filtergraph (via lenses, folding mirrors, interference filters, polarimeter). The speckle reconstruction according to Eq. (1) cannot account for this deterioration since the focus of the broadband speckle images is located in front of the narrowband channel, on a different optical path. The broadband images possess a spatial resolution certainly better than 0. '3. A spatial resolution of the narrowband continuum images of better than $\left(0.3^{2}+0.4^{2}\right)^{1 / 2}$ arcsec $=0.5$ follows. The line core image in Fig. 1 has a similar quality.

To further improve the resolution, we deconvolved the narrowband continuum and line core images at $6301 \AA$ with a Gaussian point spread function of 0.35 FWHM, applying a Tikhonov regularisation. The width of 0.35 was chosen conservatively to keep noise at a low level. This yields a spatial resolution of the narrowband images better than 0.35 . The de-convolved images of the limb and centre side parts of the sunspot NOAA9919 are collected together with the specklereconstructed images in Fig. 3.

One notices that at scales larger than 0.5 the structures, or conglomerates of structures, retain their identity through the atmospheric part covered by the observations. Yet, very importantly, when moving from low to high layers, penumbral fibrils with diameters of $0 . ' 4$ and less tend to change position, orientation, length and brightness relative to other ambient fine structures. Close inspection of Fig. 3 shows that some of the very fine structures are visible only in one of the two images originating from two different atmospheric heights. At scales below 0.' 4 the penumbral intensity fine structure changes with height in the atmosphere. Distinct examples are contained in a sub-image of Fig. 3 discussed in detail below together with magnetic field and velocity fine structures (cf. Fig. 6).

\subsection{Magnetic fields and velocities}

\subsubsection{Fe II $6149 \AA$ observations at $\theta=23^{\circ}$}

We present in Fig. 4 the speckle-reconstructed images, the effective magnetic fields $B_{\text {eff }}$ and the velocities in the sunspot 

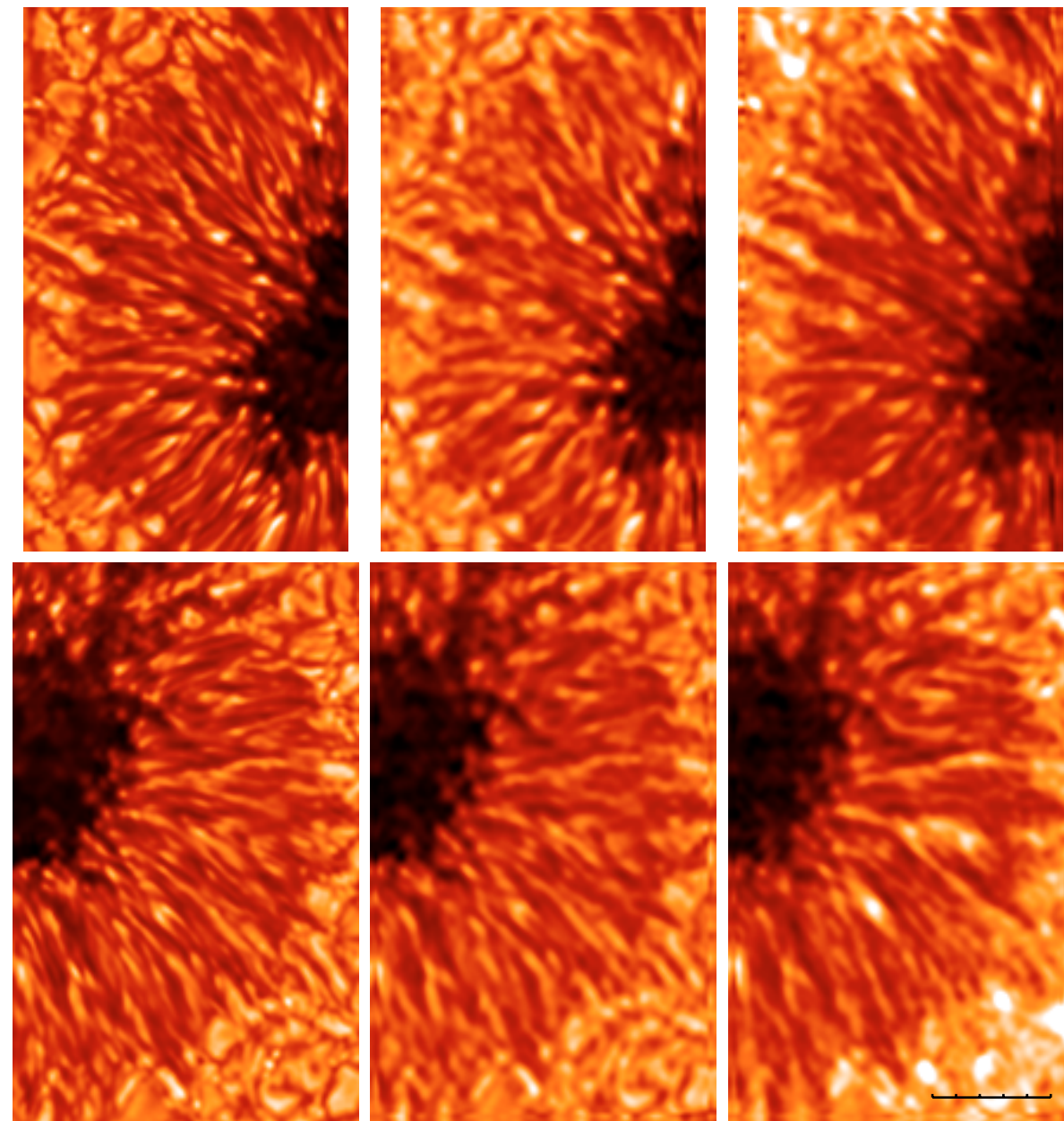

Fig. 3. Reconstructed images of the sunspot NOAA9919 on 29.04 .2002 in the $6301 \AA$ spectral range. Top: limb side part, bottom: centre side; from left to right: broadband images, narrowband images after deconvolution from the outer wings ("continuum"), and from the line core. The distance of the tickmarks is $1^{\prime \prime}$.
NOAA0346 on 2. May 2003, obtained with the COG method in the Fe II $6149 \AA$ line. The WFA for magnetic field determinations gave consistent results. Because of its origin from ionised iron, this Fe II line is very sensitive to the temperatures prevailing in the penumbra, where it is weakened compared with the ambient photosphere, and it should not occur in the umbra, where its signal was indeed very low. We find from spectral line modeling that its line centre in the penumbra is formed at heights between $z=-20 \mathrm{~km}$ and $z=+120 \mathrm{~km}$ (above $\tau_{5}=1$ ).

Before discussing details we notice the general properties of the maps:

1. In both the magnetograms and the velocity maps the penumbral filamentary structures can be seen, despite the weakness of the Fe II $6149 \AA$ line. At the outer penumbra, the magnetic signals are often too low for a reliable measurement. The dark, dot-like structures near the umbra in the magnetograms of Fig. 4 are discussed in the list below under item 4 . Beyond these, the grainy structure with scales of $0.2-0 . ' 4$ arises from noise due to the weakness of the magnetic signals in this Fe II line.

2. The magnetic field is oriented towards the observer at the disc-centre side of the penumbra and away from the observer in some parts of the limb-side penumbra.

3. Likewise, the Evershed flow is clearly seen, i.e. as velocities towards (blue shifts) and away from (red shifts) the observer in the centre and limb side penumbra, respectively.
A close inspection of the maps in Fig. 4 reveals the following special details:

1. The magnetic fields oriented towards the limb (indicated by negative field strengths in the magnetograms, denoted by " 1 " in Fig. 4) at the limb side penumbra can be interpreted as the more horizontal fields of the un-combed magnetic structuring. At positions where they intrude into the umbra, they are located between bright elongated structures. This behaviour continues outwards: more horizontal fields coincide with dark fibrils or with diffuse structures.

2. We refer to the long magnetic, filamentary structure close to feature " 1 " with $B$ oriented towards the observer and intruding into the umbra. It is denoted by " 2 " in Fig. 4. From its positive polarity, we interpret it as a structure with small magnetic field inclination (with respect to the vertical to the solar surface) in the picture of un-combed fields. The lower sharp border of the magnetic feature is located close to strong outflows and follows the border of a dark filament all along its path from the umbra to the outer penumbra. Concerning intensities, the magnetic feature houses bright and dark structures. Thus, we cannot tell conclusively whether fields with small inclination are located in bright or dark structures.

3. In the upper left part of the umbra one notices two bright filaments intruding into the umbra ("3" in Fig. 4). The magnetic field component along the LOS is strongest at their 

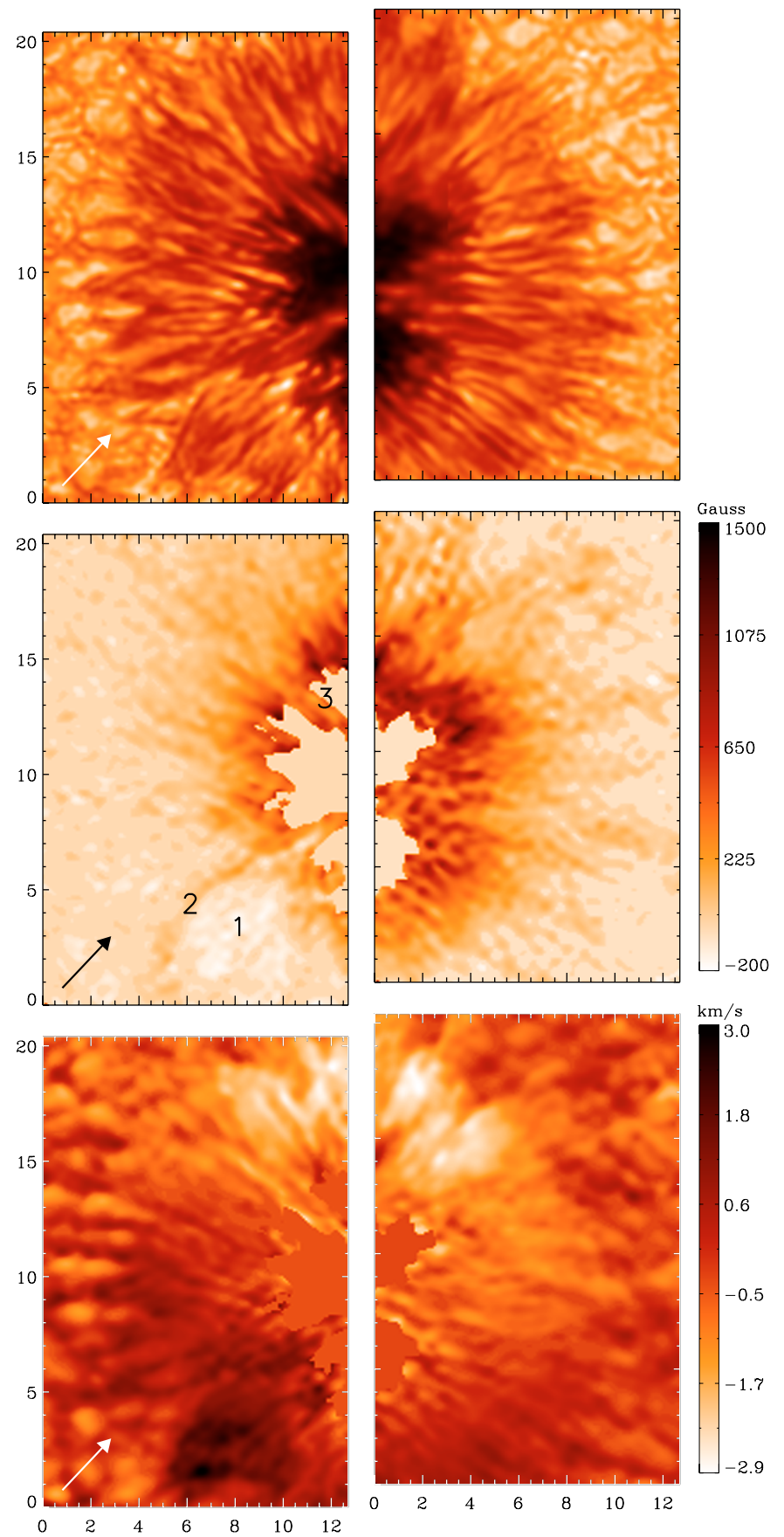

Fig. 4. Speckle-reconstructed broadband images of the sunspot NOAA0346 (upper panels), its magnetic field strengths $B_{\text {eff }}$ (middle panels) and velocities (lower panels) determined in Fe II 6149 with the COG method. The magnetic field and velocity maps are padded with an average shade when the signals were too low for a reliable determination (for $B_{\text {eff }}$ less than 50 Gauss). Velocities away from the observer are positive and are coded with dark color. The arrow points towards disc centre and the coordinates are in arcsec.

ends and between them. This supports again the moving flux tube model with the most vertical magnetic fields at the emerging point bending outwards into a more horizontal direction. Also, the un-combed field picture is confirmed if one interpretes the observation of strong LOS field components between the two bright filaments as a signature of more vertical magnetic fields. Generally, near the umbra, the magnetic filaments with strong LOS components are located in dark structures. This however contradicts in some sense item 1 above, where the "horizontal" magnetic field is also located between two bright filaments. Before entering into an interpretation of this contradiction, we prefer to perform (forward) modeling with adequate viewing angles and with adequate geometry of the magnetic fields in various thermal structures, which is deferred to a follow-up study.

4. Strong LOS magnetic field components near the border to the umbra are seen as dark dots in the magnetograms of Fig. 4. They are located at the ends of diffuse bright features, not at the bright penumbral grains in the intensity maps. We may identify them with the umbral ends of emerging flux tubes in the model by Schlichenmaier et al. (1998). In this model, the magnetic field of the tube has the lowest inclination (with respect to the vertical) and the gas inside the tube is hottest at the umbral border.

5. On the disc-centre side, near the border between the penumbra and the quiet photosphere, the picture is less conclusive. The magnetic filaments with strong LOS components may coincide with dark filaments, or with more diffuse or bright structures.

6. The filamentary structuring of the velocity field is best seen near a dividing line across the sunspot perpendicular to the limb-to-centre direction. There, a rapid change of the LOS velocity occurs. On the disc-centre side, blue-shift occurs both in bright structures and in their outward tails. Yet not every bright structure contains flows, and the highest velocities do not occur in the brightest filaments. This behavior is seen throughout the penumbra, from near the umbra to the border to the normal photosphere. In contrast to the centre side, the positions of the outward flows on the limb side exhibit a systematic difference: there, the LOS velocities are generally largest in dark structures. Our interpretation is that the strongest outflows are concentrated in more "horizontal" magnetic fields. Compared with the ambient, more vertical background field, they occur at positions with strong LOS magnetic components at the limb side and at positions with weak LOS magnetic field components on the centre side. The more vertical background field on the limb side has only a small LOS component. But this LOS component is larger than the LOS component of the horizontal field on the centre side. The LOS velocities can attain values of $3 \mathrm{~km} \mathrm{~s}^{-1}$ and thus, accounting for the projection, the velocities may be in the range of $5-7 \mathrm{~km} \mathrm{~s}^{-1}$.

7. The pattern of high LOS velocities consists of more short, only a few arcsec long features with a knotted appearance rather than of elongated fibrils, which is especially well seen in the limb side penumbra.

The inconclusiveness of earlier observational results on the relation between intensity and Evershed flow (item 6 above) thus appears to be an outcome of the viewing angle, i.e. from which side, centre or limb side, the analysed data were taken. The explanation of the systematic difference, that the higher LOS velocities occur at bright features on the centre side and at dark 
features on the limb side, can again be found with the picture of the moving flux tube in mind: the upflows of hot gas that are seen on the centre side are more vertical. Once the gas has cooled and the magnetic tube has bent into the horizontal direction, the flow will be seen best on the limb side. An extension of this model can also explain the shortness of the high-speed LOS flow features (item 7 above). Following Schlichenmaier (2003) it appears reasonable to assume downward bending of the flow channels towards subphotospheric layers within short distances, as had been found by Schlichenmaier \& Schmidt (2000). The magnetic flux tubes carrying the flows then resemble then "sea serpents" (Schmidt 2003).

\subsubsection{Fe I 6301 and $6302 \AA$ observations at $\theta=20^{\circ}$}

We now turn to the relations between (speckle reconstructed) broadband intensities, magnetic fields and velocities measured in the Fe I $6302.5 \AA$ line. These observations possess a still better spatial resolution than the ones of Fe II 6149 described above. Also, Fe I 6302.5 is more sensitive to magnetic fields and makes it possible to determine (within the approximations discussed in Sect. 2.4) the amplitude and polarity of the magnetic fields from the separation of the $V$ extrema as well as the LOS components from COG. It is formed higher in the atmosphere than Fe II 6149 and thus reflects different atmospheric properties. Figure 5 shows the relevant images of both halves of NOAA9919, the broadband images (upper left), the velocity maps from COG (lower left) and the magnetograms for the LOS components ( $B_{\text {eff }}$ ) from COG (upper right) and from the separation of the $V$ extrema (lower right).

The narrowband images reconstructed according to Eq. (1), showed only little noise. Therefore, before calculating velocity maps and magnetograms, an additional deconvolution has been applied to them as discussed in Sect. 3.1 and demonstrated in Figs. 1 and 3. By close inspection, we find the following properties:

1. The LOS magnetic field calculated from COG (upper right panels of Fig. 5) shows an inversion of polarity at the limb side where one sees negative magnetic field filaments in the middle and outer penumbra (apart from small-scale negative polarity fields in the ambient "quiet" photosphere). They are intersected by positive polarity filaments. The negative fields have LOS components with extremal values of -300 Gauss, and coincide with dark intensity structures in the continuum (or broadband) images. The positions of negative polarity fields, i.e. of their LOS components, at the limb side in dark intensity structures coincide with the outward velocity described in item 5 below.

2. Again on the limb side, strong LOS magnetic fields of 1500-2500 Gauss are found in dark intensity filaments, yet not in their darkest parts but rather displaced to the borders to bright structures. We recall the similar results from the 6149 observations (item 3 in Sect. 3.2.1). In any case, on the limb side, strong LOS magnetic field components are not found in bright structures. These certainly contain also magnetic fields, but with weaker LOS components.
3. In the centre-side penumbra, the strong LOS magnetic field components are located in dark intensity structures, or in diffuse intensity patterns.

4. Negative polarities are also found from the $V$ amplitude separations, but not in such fine detail as from the COG method. When comparing the LOS components with the field strengths determined from the separation of the $V$ extrema we notice that close to the line perpendicular to the limb-to-centre direction and crossing the centre of the umbra the fluctuations of the field amplitude and of the LOS component coincide spatially. At locations towards the limb, this coincidence is mostly preserved, but not always. However, surprisingly, at locations towards the disc centre there exists an anti-correlation between fluctuations of the field amplitude and of the LOS component. A possible explanation lies in (anti-)correlated fluctuations of the field strength and the field inclination (with respect to the vertical) which have no influence at positions of the line perpendicular to the limb-to-centre direction and are less perceptible on the limb side of the penumbra. The anticorrelated fluctuations of field strength and field inclination were already found, from lower spatial resolution data, by Lites et al. (1993) and Westendorp Plaza et al. (2001a) and were discussed by Martínez Pillet (1997). A simulation of Stokes profiles in a forthcoming study will take up this finding.

5. Consistently with the finding for the Fe II 6149 observations, the outward Evershed flow on the disc-centre side occurs at positions of bright filaments in the continuum images or at their somewhat weakened tails, while on the limb side the outward flow is definitely located in dark structures. The reverse is not true: not all bright structures (on the centre side) exhibit strong outflows.

6. The outward velocities, i.e. their LOS components, are smaller than those found in Fe II 6149, they rarely attain values larger than $1.5 \mathrm{~km} \mathrm{~s}^{-1}$. Only a small part of this difference can be ascribed to observation at a smaller heliocentric angle, i.e. at $20^{\circ}$ compared to $23^{\circ}$ for the $6149 \mathrm{ob}$ servations, or because the data are from different sunspots. The difference reflects the often reported decrease of the Evershed flow with height (e.g. Börner \& Kneer 1992, and references therein).

7. The relation between velocities and intensities from the line core of 6301.5 is less clear. On the centre side, large velocities can occur where the line core intensity is less bright than the neighborhood. This likely reflects the fact that the information about the velocities, measured from COG in 6302.5, stems from deeper layers than the 6301.5 line core intensities.

8. The positions of the Evershed flow and the strong LOS magnetic field components, close to each other, but not fully coinciding, in dark intensity filaments at the limb side, discussed in items 2 and 5 above, are not inconsistent. The flow channels are likely smaller than the spatial resolution of our observations and strong, more vertical magnetic background fields need not necessarily coincide with them. On the limb side, the velocities are perceptible only when the flow channels are bent into a more horizontal direction 

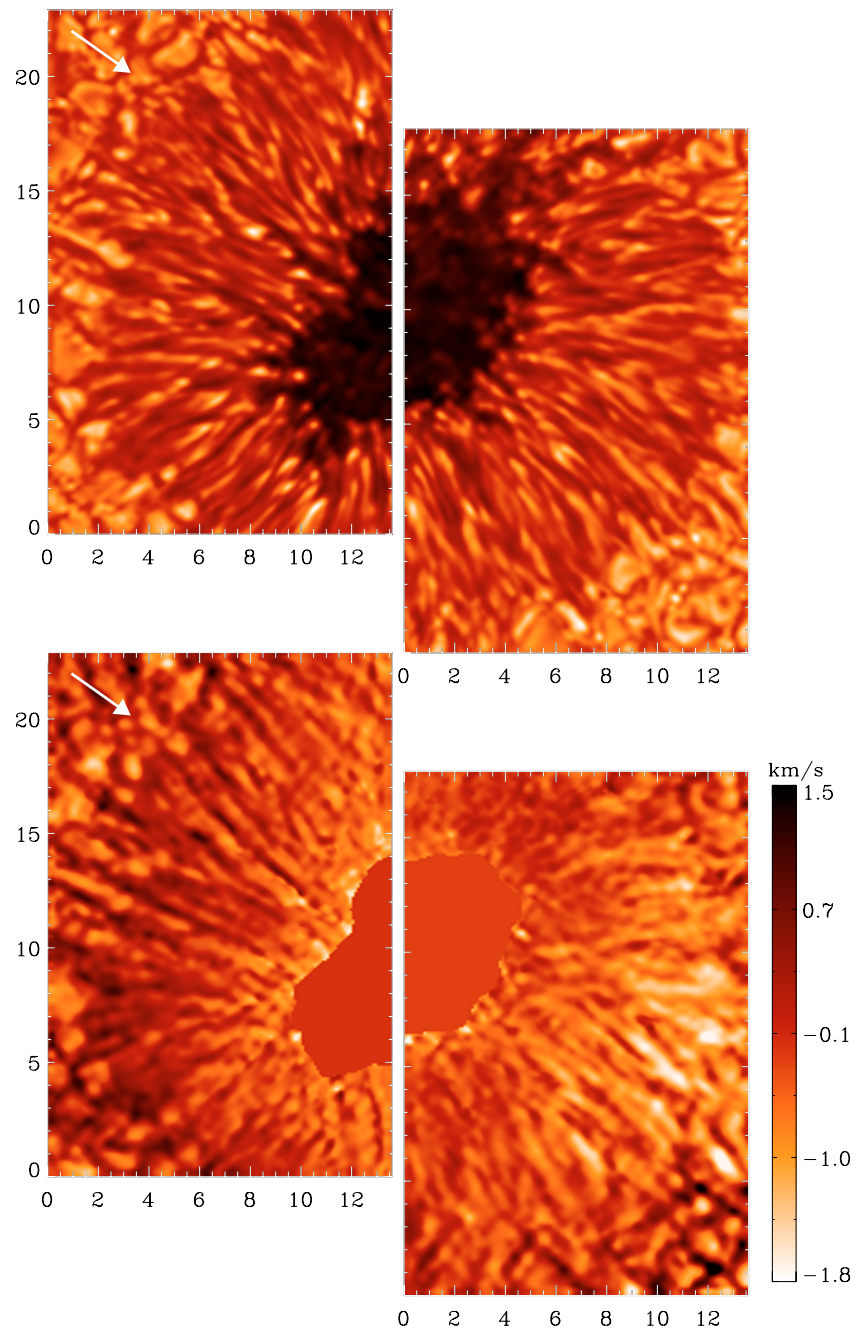
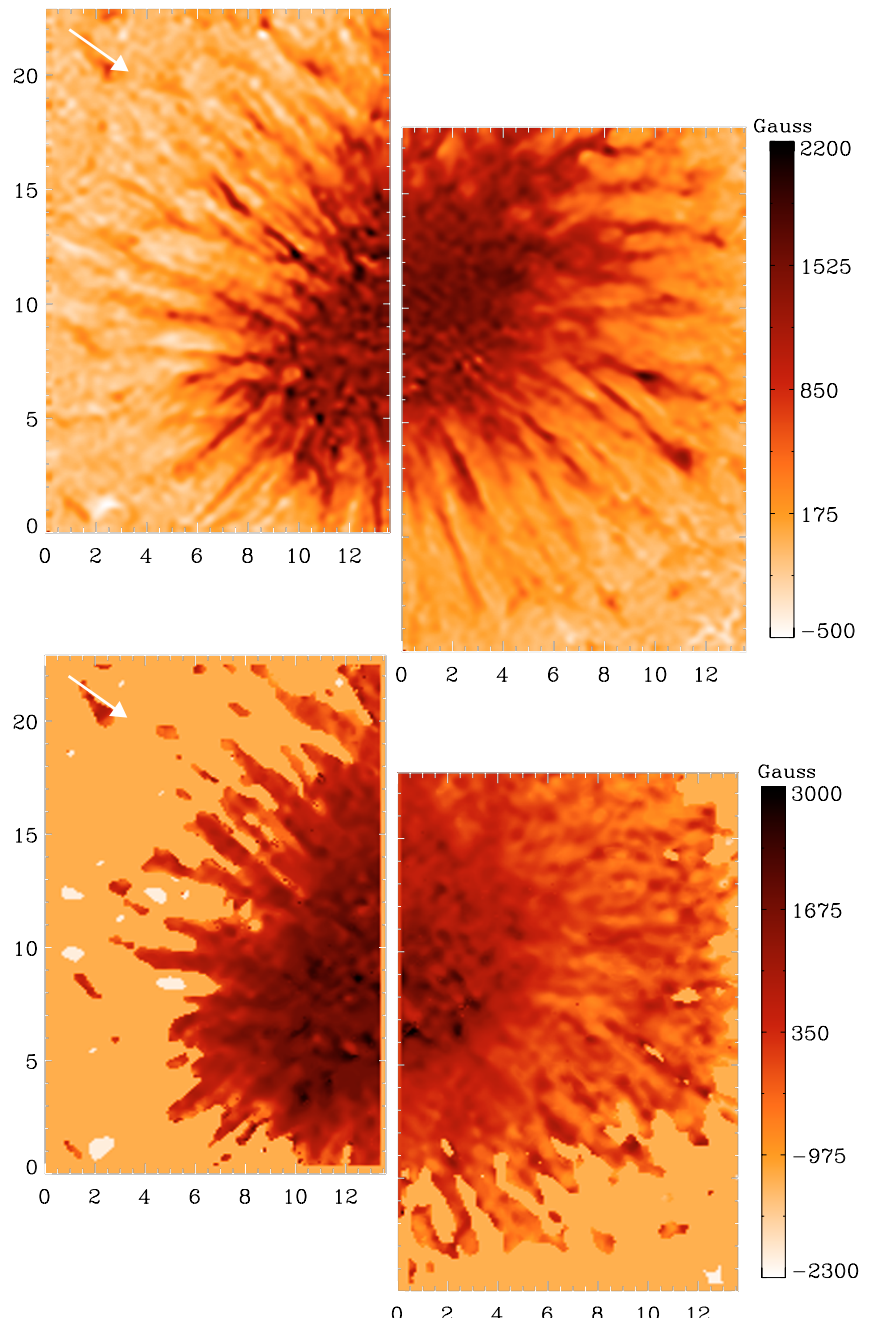

Fig. 5. Speckle reconstructed broadband images of the sunspot NOAA9919 (upper left panels), its velocities from COG (lower left panels) and magnetic field strengths $B_{\text {eff }}$ from COG (upper right panels) and from the separation of $V$ extrema (lower right) in Fe I 6302.5. The magnetogram from the $V$ amplitude separation is padded with zero shade when the signals are too low for a reliable determination. Velocities away from the observer are positive and are coded with dark color and velocity values in the umbral area are set to zero because there the COG method does not yield reliable values. The arrow points towards disc centre and the coordinates are in arcsec.

ore even downward, when the gas, originally hot and rising, has cooled down.

In general, our high-spatial-resolution data confirm the uncombed magnetic field structure of penumbrae. Title et al. (1993) and Bellot Rubio et al. (2003), among others, found that the more horizontal flux tubes carry the Evershed flow. The latter authors applied inversion techniques to full Stokes profiles of the magnetically sensitive infrared Fe I lines at $1.565 \mu \mathrm{m}$. Their data had lower spatial resolution than the ones presented here and the authors concluded indirectly concerning the magnetic field and velocity structure by adopting two-component models for the inversions. Our high-resolution data are compatible with this picture.

We show in Fig. 6 a subfield of the disc centre side of the penumbra in Fig. 5 to discuss some special dynamical features.

1. Feature "1", located in the middle penumbra is bright in the 6301.5 line core, but not very brilliant in the continuum/broadband image. A strong magnetic field of approximately 2000 Gauss is found, consistently from the $V$ separation and from COG. The magnetic field is thus oriented close to the LOS direction. At the position of the strong field a downward velocity into the dark structure in the continuum image, with a LOS component away from the observer of approximately $0.5 \mathrm{~km} \mathrm{~s}^{-1}$, is seen. This behaviour, strong magnetic fields in features which are relatively dark in the continuum and bright in the line core, at positions of downflows, is different from the general relation between magnetic fields and velocities (no gas flow in strong field features). It demonstrates the richness of penumbral processes which may still reveal unexpected properties.

2. Feature "2" contains a magnetic filament with an amplitude of the LOS component of 1500 Gauss. It ends near the outer penumbra in a relatively dark structure in the continuum image. Along the magnetic filament, the relation with continuum intensity is not straight, the filament coincides at some places with more bright and at other positions with 


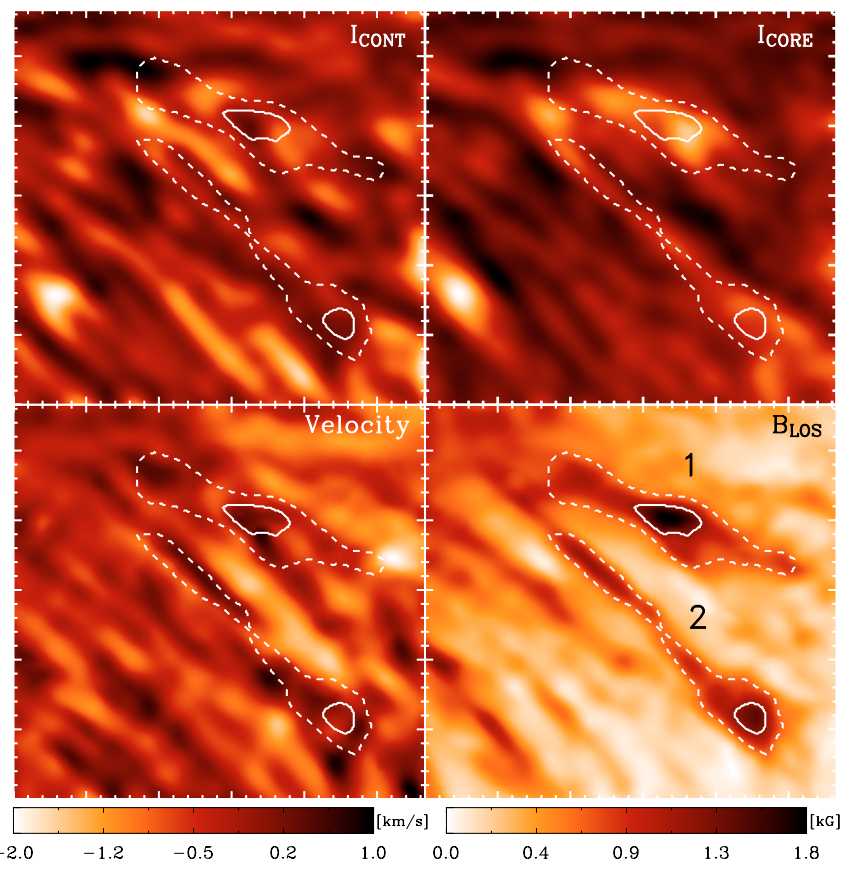

Fig. 6. Subfield of the disc centre side of the penumbra in Fig. 5 with continuum image (upper left), 6301.5 line core image (upper right), velocity map (lower left) and magnetogram from COG (lower right). Magnetic field contours (solid) at a level of 1200 Gauss and contours surrounding magnetic features (dashed) are overlaid. The longer tickmarks are at $1^{\prime \prime}$ distance.

more dark features. But in the 6301.5 line core intensity a bright structure is at the same position as the magnetic filament. The velocity shows a down-flow at the filament's start in the inner penumbra, which is interrupted along the filament and exhibits again strong down-flows of $0.5 \mathrm{~km} \mathrm{~s}^{-1}$ surrounding its end in the outer penumbra. Yet the strongest magnetic field at this end coincides with an up-flow of approximately $0.5 \mathrm{~km} \mathrm{~s}^{-1}$.

3. Figure 6 demonstrates the variation in the appearance of structures with scales of 0.5 and smaller, at different heights. Many of the fibrils, seen to be bright in the continuum image, have lost their brilliance in the line core image (from $300 \mathrm{~km}$ ), some are shortened or have disappeared. Some other structures are relatively enhanced. As an example, at the upper left ends between feature " 1 " and feature " 2 " in Fig. 6 a bright filament, oriented towards the lower right, is seen in the continuum image. In the line core image, this filament is shorter and dark at its lower right end. Further examples can easily be found, e.g. in the lower left corner of this subfield.

\section{Conclusions}

Spectropolarimetric observations from penumbrae in the Fe II $6149 \AA$ line and in the Fe I $6302 \AA$ line pair were analysed. By means of image reconstruction we achieved a spatial resolution of the spectroscopic data of 0.5 and better. The data in the two spectral regions were obtained for two different sunspots. Yet we may assume that the typical penumbral dynamics are the same in more or less symmetric sunspots. The Fe II line is formed deeper in the atmosphere than the Fe I line pair and thus gives information about deep layers. We summarise our main results:

1. On scales larger than $0{ }^{\prime} 5$ the intensity pattern of penumbrae does not change when seen in radiation originating from either deep photospheric or higher layers $(300 \mathrm{~km})$. Yet at scales of 0.5 and smaller the patterns in the two atmospheric ranges are clearly different. The structuring is height-dependent with vertical extents of the same scale as the horizontal thickness of the fibrils.

2. Interestingly, the small-scale channels of the Evershed flow coincide with different features on the limb and the disccentre side of the sunspots. This can be understood in the picture of rising flux tubes of Schlichenmaier et al. (1998). It appears that at the limb side the flow is best seen when the originally hot, rising gas has cooled down and the motion has turned horizontal or even downward. On the centre side, the flow is best seen in bright, rising structures. The asymmetry of the visibility of flows between centre and limb side was also seen in line bisectors by Schlichenmaier et al. (2004). This result from high-spatial-resolution observations confirms and specifies in more detail findings by Westendorp Plaza et al. (2001b). These authors also find, from lower resolution data, a visibility difference of velocities between limb and centre side and interprete this as an opacity effect. From correlations between field inclination, field strength and velocity, they conlude that the Evershed channels are mostly located in the space between the "spines" and carry cooler material.

3. Strong line-of-sight components of the magnetic fields are not found in bright filaments but in dark structures, somewhat displaced from the darkest parts, in both the limb and the centre side penumbrae. On the limb side, their positions do not coincide with those of the strongest velocity fields.

The observations in Fe II $6149 \AA$ and in Fe I $6302 \AA$ were obtained from two different sunspots. Yet the analysis of the two data sets gave a consistent picture. Generally, our findings confirm the un-combed magnetic field structure. They are also compatible with the picture of low lying magnetic flux tubes, which possibly even submerge back within the penumbra. This model was derived from low spatial resolution data, but with full polarimetric information by Bellot Rubio et al. (2003) assuming two-component atmospheres within the spatial resolution element.

The success of our analysis relies on both the twodimensionality and the high spatial resolution of the spectropolarimetric information. Without two-dimensionality one would not see the differences between limb and centre side behaviour of intensity, velocity and magnetic fields and their relations. Also, it was possible only with sufficient spatial resolution to see in detail these relations and to detect the differences between limb and centre side.

Many aspects of penumbrae have remained inconclusive. One cannot define strict rules for relations between intensities from various atmospheric levels, velocities and magnetic fields. There are special cases which apparently contradict the clear picture of flow channels with low magnetic fields. Also, high 
intensity in continuum or spectral line cores is not a matter of velocity or magnetic field, but primarily of temperature which itself also depends on the state of the ambient gas and on the history of the feature. Thus, bright does not necessarily mean weak (or strong) magnetic field.

Only the intensity measurements, i.e. $I_{\lambda}$, were used for height discrimination of the intensity structure. It will be important to find, by forward modelling, height dependences of magnetic field and velocity structures compatible with the observations and simultaneously with the basic laws governing magnetic fields and gas flows.

Acknowledgements. The authors thank Dr. A. Hofmann for providing the data of the VTT instrumental polarisation. The referee is thanked for the constructive criticisms. N.B.G. gratefully acknowledges a fellowship of the Graduiertenkolleg GRK 140 Strömungsinstabilitäten und Turbulenz by the Deutsche Forschungsgemeinschaft (DFG) and IDC acknowledges a fellowship from DFG through grant 418 SPA112/14/01. O.V.O. thanks the German Academic Exchange Service - DAAD for support through grant A/00/01395 and K.G.P. thanks the DFG for support through grant KN 152/29-1. The Vacuum Tower Telescope is operated by the Kiepenheuer-Institut für Sonnenphysik, Freiburg, at the Spanish Observatorio del Teide of the Instituto de Astrofîsica de Canarias on Tenerife.

\section{References}

Balthasar, H., Schmidt, W., \& Wiehr, E. 1997, Sol. Phys., 171, 331

Beckers, J. M., \& Schröter, E. H. 1969, Sol. Phys., 10, 384

Bellot Rubio, L. R. 2004, Rev. Mod. Astron., 17, 21

Bellot Rubio, L. R., Balthasar, H., Collados, M., \& Schlichenmaier, R. 2003, A\&A, 403, L47

Bendlin, C., \& Volkmer, R. 1995, A\&AS, 112, 371

Bendlin, C., Volkmer, R., \& Kneer, F. 1992, A\&A, 257, 817

Börner, P., \& Kneer, F. 1992, A\&A, 259, 307

Capitani, C., Landi degl'Innocenti, E., Cavallini, F., Ceppatelli, G., \& Landi degl'Innocenti, M. 1989, Sol. Phys., 120, 173

Collados, M. 1999, in Third Advances in Solar Physics Euroconference: Magnetic Fields and Oscillations, ed. B. Schmieder, A. Hofmann, \& J. Staude, ASP Conf. Ser., 184, 3

de Boer, C. R. 1996, A\&AS, 120, 195

de Boer, C. R., \& Kneer, F. 1992, A\&A, 264, L24

Degenhardt, D., \& Wiehr, E. 1991, A\&A, 252, 821

Degenhardt, D., \& Wiehr, E. 1994, A\&A, 287, 620

del Toro Iniesta, J. C., Tarbell, T. D., \& Ruiz Cobo, B. 1994, ApJ, 436, 400

Eibe, M. T., Mein, P., Roudier, T., \& Faurobert, M. 2001, A\&A, 371, 1128

Evershed, J. 1909, MNRAS, 69, 454

Hirzberger, J., \& Kneer, F. 2001, A\&A, 378, 1078

Hirzberger, J., Koschinsky, M., Kneer, F., \& Ritter, C. 2001, A\&A, 367,1011

Hofmann, A. 2001, private communication
Janßen, K. 2003, Ph.D. Thesis, Göttingen university

Keller, C. U., \& von der Lühe, O. 1992, A\&A, 261, 321

Koschinsky, M., Kneer, F., \& Hirzberger, J. 2001, A\&A, 365, 588

Krieg, J., Wunnenberg, M., Kneer, F., Koschinsky, M., \& Ritter, C. 1999, A\&A, 343, 983

Landi Degl'Innocenti, E. 1992, in Solar Observations: Techniches and interpretations, ed. F. Sánchez, M. Collados, \& M. Vázquez (Cambridge: Cambridge University Press), 71

Lites, B. W., Skumanich, A., \& Scharmer, G. B. 1990, ApJ, 335, 329

Lites, B. W., Elmore, D. F., Seagreaves, P., \& Skumanich, A. P. 1993 , ApJ, 418, 928

Martínez Pillet, V. 1997, in First Advances in Solar Physics Euroconference: Advances in Physics of Sunspots, ed. B. Schmieder, J. C. del Toro Iniesta, \& M. Vázquez, ASP Conf. Ser., 118,212

Mein, P. 1971, Sol. Phys., 20, 3

Meyer, F., \& Schmidt, H. U. 1968, Z. Angew. Math. Mech., 48, T218

Montesinos, B., \& Thomas J. H. 1997, Nature, 390, 485

Okunev, O. V. 2004, Ph.D. Thesis, Göttingen university

Okunev, O. V., \& Kneer, F. 2004, A\&A, 425, 321

Rees, D. E., \& Semel, M. D. 1979, A\&A, 74, 1

Rimmele, T. R. 1995, A\&A, 298, 260

Rouppe van der Voort, L. H. M. 2002, A\&A, 389, 1020

Rouppe van der Voort, L. H. M., Löfdahl, M. G., Kiselman, D., \& Scharmer, G. B. 2004, A\&A, 414, 717

Scharmer, G. B., Gudiksen, B. V., Kiselman, D., Löfdahl, M. G., \& Rouppe van der Voort, L. H. M. 2002, Nature, 420, 151

Schlichenmaier, R. 2003, in Current Theoretical Models and Future High Resolution Observations, ed. A. A. Pevtsov, \& H. Uitenbroek, ASP Conf. Ser., 286, 211

Schlichenmaier, R., \& Schmidt, W. 2000, A\&A, 358, 1122

Schlichenmaier, R., Jahn, K., \& Schmidt, H. U. 1998, A\&A, 337, 897

Schlichenmaier, R., Bellot Rubio, L. R., \& Tritschler, A. 2004, A\&A, 415,731

Schmidt, W. 2003, Astron. Nachr., 324, 374

Semel, M. D. 1967, Ann. Astrophys., 30, 513

Solanki, S. K. 2003, A\&AR, 11, 153

Solanki, S. K., \& Montavon, C. A. P. 1993, A\&A, 275, 283

Stanchfield, D. C. H., Thomas, J. H., \& Lites, B. W. 1997, ApJ, 477, 485

Stellmacher, G., \& Wiehr, E. 1981, A\&A, 103, 211

Title, A. M., Frank, Z. A., Shine, R. A., et al. 1993, ApJ, 403, 780

Tritschler, A., Schlichenmaier, R., Bellot Rubio, L. R., \& the KAOS Team 2004, A\&A, 415, 717

von der Lühe, O. 1984, J. Opt. Soc. Am. A1, 510

Weigelt, G. P. 1977, Opt. Commun., 21, 55

Westendorp Plaza, C., del Toro Iniesta, J. C., Ruiz Cobo, B., et al. 2001a, ApJ, 547, 1130

Westendorp Plaza, C., del Toro Iniesta, J. C., Ruiz Cobo, B., \& Martínez Pillet, V. 2001b, ApJ, 547, 1148

Wiehr, E. 1999, in Third Advances in Solar Physics Euroconference: Magnetic Fields and Oscillations, ed. B. Schmieder, A. Hofmann, \& J. Staude, ASP Conf. Ser., 184, 86

Wiehr, E., \& Stellmacher, G. 1989, A\&A, 225, 528 